\title{
Eficácia da Reabilitação na mobilidade, prevenção e diminuição do risco de queda em doentes com Parkinson
}

\author{
Ricardo Loureiro', Rosa Martins², Joana Bernardo¹ e \\ Susana Batista ${ }^{3}$ \\ ${ }^{1}$ Centro Hospitalar Universitário de Coimbra, Portugal | ricardo_loureiro_1@hotmail.com; \\ joana_bernardo@live.com.pt | https://orcid.org/0000-0001-6135-2278; https://orcid.org/0000- \\ 0003-3614-9061 \\ ${ }^{2}$ Departamento de Enfermagem de Reabilitação da ESSV, Portugal | \\ rmartins.viseu@gmail.com | https://orcid.org/0000-0001-9850-9822 \\ ${ }^{3}$ Departamento de Enfermagem de Reabilitação da ESSV, Portugal | \\ susanapbatista@gmail.com | https://orcid.org/0000-0003-0256-6027
}

\begin{abstract}
Resumo: Introdução: O doente com Parkinson apresenta frequentemente alterações na postura, diminuição da mobilidade e um maior risco de incidência de quedas. Objetivos: Avaliar a eficácia de programas de Reabilitação na melhoria da mobilidade, na prevenção e diminuição do risco de queda em doentes com Parkinson.Métodos: Utilizando a estratégia PICO, realizou-se uma Revisão Integrativa no período temporal 2012-2019. O corpus do estudo integrou 7 estudos primários, resultantes de pesquisa em bases de dados nacionais e internacionais. Seguiram-se os princípios propostos pela Cochrane Handbook. Resultados: Ficou demonstrada a eficácia dos planos de Reabilitação na melhoria da mobilidade e no risco de queda dos doentes. Os protocolos de intervenção de Enfermagem de Reabilitação devem incluir para além dos exercícios clássicos (treinos de marcha, de equilíbrio e correção postural), exercícios de estimulação propriocetiva, recurso à Realidade Virtual e intervenções tecnológicas. Conclusões: Os programas de Reabilitação instituídos nos doentes com Parkinson mostraram-se eficazes, contudo a introdução de novas estratégias de intervenção pode aumentar significativamente melhorias na saúde dos doentes.
\end{abstract}

Palavras-chave: Doença de Parkinson; Imobilização; Acidentes por Quedas; Enfermagem em Reabilitação.

Efficacy of Rehabilitation on Mobility, Prevention and Reduction of the Fall's Risk in Parkinson's Patients

Abstract: Introduction: The patient with Parkinson disease often presents changes in posture, decreased mobility and an increased risk of falling. Objectives: To evaluate the effectiveness of Rehabilitation programs in improving mobility, preventing and decreasing the risk of falling in Parkinson's patients. Methods: Using the PICO strategy, an Integrative Review was carried out in the period 2012-2019. The study corpus included 7 primary studies, resulting from research in national and international databases. The principles proposed by the Cochrane Handbook were followed.Results: The effectiveness of Rehabilitation plans in improving mobility and the fall's risk for patients was demonstrated. The Rehabilitation Nursing intervention protocols must include, in addition to the classic exercises (gait, balance and postural correction training), proprioceptive stimulation exercises, use of Virtual Reality and technological interventions. Conclusions: The Rehabilitation programs instituted in patients with Parkinson's disease demonstrated to be effective, however the introduction of new intervention strategies can significantly improve the patients' health.

Keywords: Parkinson Disease; Immobilization; Accidental Falls; Rehabilitation Nursing.

\section{Introdução}

O processo de envelhecimento humano exige atualmente uma particular atenção pelas Ciências da Saúde. Trata-se de um processo, que tende a ser cada vez mais longo e simultaneamente acompanhado por alterações fisiológicas e biológicas associadas a processos degenerativos, cujas implicações negativas se fazem sentir sobretudo na capacidade funcional das pessoas (Rocha et al. 2017; Martins et al. 2018). 
A doença de Parkinson (DP), no leque das doenças progressivas e degenerativas do Sistema Nervoso Central, é das mais comuns, ocupando em Portugal o segundo lugar. Dados recentes do Instituto Nacional de Estatística (INE, 2019) mostram que em Portugal, no ano de 2017, se registaram 935 mortes devido à DP, representando $0,8 \%$ da mortalidade no nosso país. À semelhança dos valores epidemiológicos a nível mundial, os homens apresentam uma maior taxa de mortalidade em relação às mulheres, com $0,9 \%$ do total de óbitos e $0,8 \%$ do total de óbitos em mulheres. E ainda segundo a Associação Portuguesa de Doentes de Parkinson (APDP, 2019), estima-se que em Portugal possam ser afetadas por esta doença entre 18000 a 20000 pessoas. A idade parece constituir-se como um fator de risco importante, uma vez que, cerca de $97 \%$ dos óbitos por DP ocorreram em pessoas com 65 e mais anos, e com uma percentagem de $85 \%$ em pessoas com 75 e mais anos. Dados do INE (2019) destacam ainda uma idade média de 82,7 anos nos óbitos por esta patologia.

A DP é, na perspetiva apresentada pela APDP (2019), uma doença crónica que afeta o sistema motor, ou seja, que envolve os movimentos corporais, levando a tremores, rigidez, bradicinesia, instabilidade postural e alterações da marcha. A instabilidade postural, associada à perda de reflexos posturais, provoca repercussões muito negativas no equilíbrio levando a um aumento muito significativo das quedas nestes pacientes (Santos, 2018). As quedas, constituem de facto uma situação comum nestes doentes, apresentando uma incidência que varia entre os 40 e os $70 \%$, valores estes bastante superiores aos verificados em outras patologias neurológicas (Nielsen, Santos, Gering, \& Sant'Ana, 2018). Embora se trate, de uma patologia reconhecida internacionalmente como doença do movimento, são vários os sintomas não motores que podem surgir, envolvendo múltiplas funções, como o ciclo de regulação sono-vigília, componente cognitiva e comportamental, humor, alterações específicas do sistema nervoso autónomo, funções sensoriais e de perceção da dor (Barbosa \& Melo, 2017). Além do referido, condiciona a funcionalidade, a autonomia, a independência e a qualidade de vida, quer da pessoa portadora da doença, quer da própria família. Pelas razões apontadas, o controle e alívio da sintomatologia é fundamental e representa um enorme desafio para os profissionais de Saúde, sendo atualmente discutida pela comunidade científica, a importância de uma intervenção de Reabilitação multidisciplinar. Luz e Coronago (2017), dizem-nos que a terapêutica medicamentosa contribui sem dúvida para controlar os sintomas da doença, não obstante, com o passar do tempo ocorre uma perda da eficácia medicamentosa. Assim, propõem que o acompanhamento seja multidisciplinar, associando tratamento farmacológico e não farmacológico, dando especial enfoque a intervenções diferenciadas, inovadoras e integradas em programas de Reabilitação. Neste sentido e considerando as competências especializadas atribuídas aos Enfermeiros de Reabilitação (ER), cujo objetivo principal consiste em maximizar as capacidades motoras e minimizar ou atenuar as dificuldades (entre elas, a instabilidade postural e quedas) presentes no doente com Parkinson, pode trazer contributos fundamentais não só ao doente, mas também às famílias, às comunidades e à Saúde no geral (Lizon, Cholewa \& Uher, 2017).

A revisão do estado da arte sobre a matéria, demonstra que as evidências científicas nacionais e internacionais existem numa pluralidade de estudos. Muitos deles, estão direcionados para a prevenção e redução do número de quedas em pessoas com DP, e descrevem a implementação de programas de Reabilitação, contudo, encontram-se neles grandes disparidades, não só nos processos de implementação, mas também nos resultados obtidos. Estes achados, justificam a presente Revisão Integrativa (RI), que tem por objetivo avaliar a eficácia de programas de Reabilitação na melhoria da mobilidade, na prevenção e diminuição do risco de quedas em doentes com Parkinson. A opção pelas metodologias qualitativas (narrativa dos estudos) de carater mais exploratório tem a vantagem de diminuir as incertezas sobre as melhores intervenções e estabelecer prioridades e eficácia na definição e implementação dos programas de reabilitação dirigidos ás pessoas com doença de Parkinson. É ainda nosso desejo, alargar o conhecimento científico em Enfermagem (com especial enfoque na área especializada em ER) e produzir evidências que alicercem intervenções reabilitadoras integradas na prática clínica, uma vez que, são ainda escassas em Portugal. 
Deste modo e de acordo com as linhas de orientação internacional Cochrane Handbook (2017), formulou-se a pergunta de partida segundo o acrônimo PICO: Que programas de Reabilitação (I) apresentam eficácia na melhoria da mobilidade, na prevenção e diminuição do risco de quedas $(\mathrm{O})$, em doentes com Parkinson $(\mathrm{P})$ ?

\section{Métodos}

A população alvo desta RI são pessoas portadoras de DP. A pesquisa decorreu nos meses de Julho e Agosto de 2019 e foi realizada por dois autores de forma independente,no sentido de fazer validação por pares no processo de recolha de informação. Na ausência de consenso foi incluído um terceiro autor. Foi efetuada em bases de dados eletrónicas, nomeadamente: CINAHL (Cumulative Index to Nursing and Allied Health Literature) complete, Cochrane Central Register of Controlled Trials, MEDLINE (Medical Literature Analysis and Retrieval System Online) complete, por apresentarem reconhecida idoneidade científica. Complementámos ainda a nossa pesquisa com as bases de dados Pubmed (Public Medline), PEDro (Base de Dados em Evidências em Fisioterapia) e Repositórios Institucionais. No sentido de identificar evidência científica mais atualizada, definiu-se como filtro cronológico 2012-2019 e os descritores utilizados foram retirados da MeSH Browser, sendo estes: "rehabilitation; "programs" "fall's risk"; "nursing"; "Rehabilitation interventions," nos idiomas português, inglês e espanhol, com os operadores boleanos $O R$ e AND. Assim, obteve-se a fórmula: Parkinson's disease OR people AND fall AND fall's risk AND Nursing AND Rehabilitation interventions. Após a pesquisa dos estudos, foram ainda estabelecidos e aplicados critérios de seleção desses mesmos estudos, encontrando-se estes expressos no quadro 1.

Quadro 1. Critérios de seleção do estudo.

\begin{tabular}{l} 
Critérios de seleção \\
\hline Artigos publicados entre 2012 e 2019 (inclusive). \\
Artigos publicados cuja população alvo seja a pessoa com DP. São excluídos estudos que \\
simultaneamente apresentem uma ou mais comorbilidades sem relação com a doença. \\
Artigos que avaliem a eficácia de programas de Reabilitação em pessoas com DP, \\
direcionados à melhoria da mobilidade, da prevenção e diminuição do risco de queda. \\
Estudos que demonstram benefícios de programas de Reabilitação na melhoria da \\
mobilidade e/ou na prevenção e diminuição do risco de queda em pessoas com DP. \\
Artigos escritos em português, inglês e espanhol.
\end{tabular}

Posteriormente, a avaliação da qualidade dos estudos foi realizada por dois autores de forma independente, uma vez que a avaliação da sua qualidade se torna fundamental, devido às implicações na tomada de decisão na prática baseada na evidência. O Joanna Briggs Institute (JBI) propõe uma avaliação metodológica dos ensaios clínicos, que pressupõe a avaliação do risco de viés, ou seja, erros sistemáticos no desenho, condução e análise dos estudos quantitativos, que possam ter impacto na validade das inferências desses estudos (JBI, 2015). Em Portugal, Carneiro (2008), Diretor do Centro de Estudos de Medicina Baseada na Evidência (CEMBE) e da Cochrane Portugal, propõe uma grelha de análise crítica de ensaios clínicos, aos quais atribui um score de classificação final. De acordo com esta classificação, só são considerados de qualidade os estudos com pontuação igual ou superior a $75 \%$, critérios estes, aplicados nesta revisão. Dado tratar-se de uma $\mathrm{RI}$, o presente estudo não foi submetido à Comissão de Ética da Instituição de Ensino onde foi realizado, porém, todos os trabalhos utilizados foram devidamente citados e referenciados, respeitando os direitos de autor. Assim, num primeiro momento, obtivemos uma lista (com título e resumo) de 3253 artigos filtrados, que foram submetidos a uma avaliação crítica por dois revisores, de forma independente, com base na relevância do tipo de estudo, dos participantes e das intervenções para a revisão em curso. 
Após seleção dos estudos por parte de cada revisor resultou por consenso, a exclusão de 2356 artigos pelo título, e 350 por se encontrarem repetidos, ficando, portanto, 547 artigos.

Destes, após leitura de resumos, e aplicação de testes de relevância I (que consistem em estar ou não em concordância com a questão orientadora) foram excluídos 501 , ficando assim 46 artigos para prosseguir a respetiva seleção. Num segundo momento, procedeu-se à leitura integral dos 46 artigos (em texto completo) e à luz dos critérios e dos testes de relevância II, (que consistem na aplicação dos critérios de seleção), foram excluídos 41 , e selecionados apenas 7 artigos. Os valores de score total apresentados nos estudos oscilaram entre o mínimo de $84.21 \%$ (E3) e máximo de $94.74 \%$ (E5). Os procedimentos metodológicos anteriormente descritos e que compreenderam a seleção das publicações para esta RI, encontram-se ilustrados no fluxograma da figura 1.

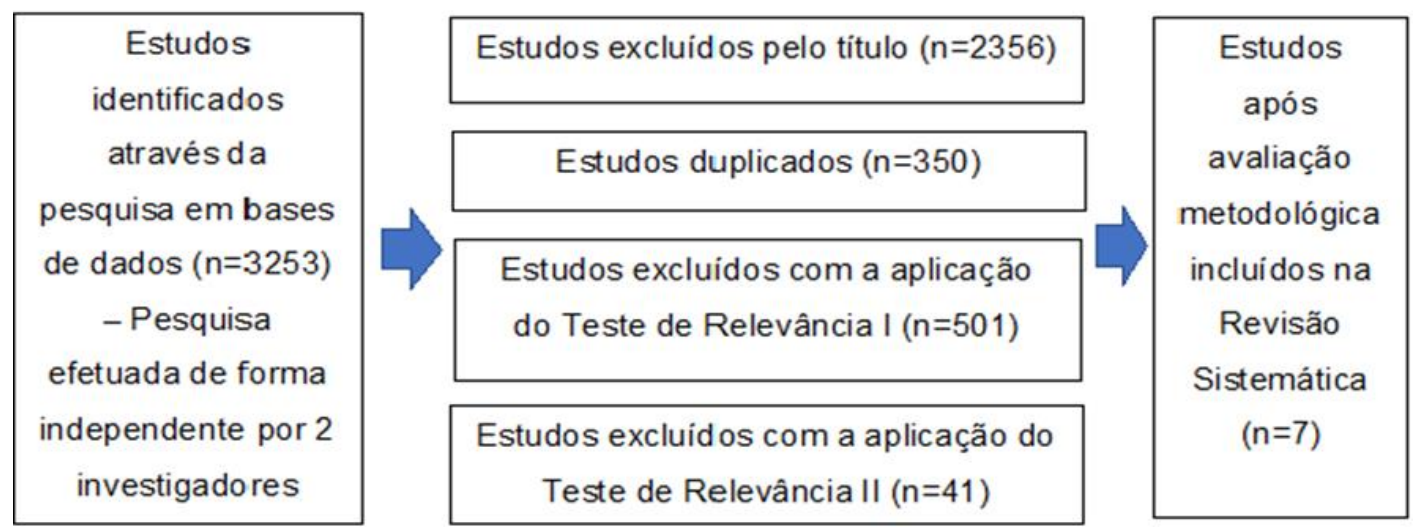

Fig. 1. Fluxograma do processo de pesquisa e seleção de estudos.

Por fim, os resultados do corpus dos artigos incluídos na Rl, foram agrupados num quadro e objeto de síntese narrativa.

\section{Resultados}

Os dados que resultaram da análise dos sete artigos que compõem o corpus deste estudo, irão ser apresentados de forma sintética no quadro 2. Por um lado, verifica-se que são estudos realizados à escala global, em diferentes zonas geográficas do Mundo, como sejam o continente Americano (América do Norte e do Sul), Europa e Ásia. Não obstante da sua localização geográfica, os estudos demarcam-se entre si pela diversidade de atualizações no que concerne à conceção de cuidados e à implementação de intervenções em Reabilitação na DP. O quadro 2 expressa informações relativas aos autores, ano de publicação e país, tipo de estudo, amostras, intervenções e sínteses dos resultados.

Quadro 2. Resumo dos estudos incluídos na revisão.

\begin{tabular}{|c|c|c|}
\hline Estudos/Autores/País & Tipo de Estudo & Participantes/Intervenções/Evidencia alcançada \\
\hline $\begin{array}{l}\text { E1 } \\
\text { Santos et al. (2012). } \\
\text { Brasil }\end{array}$ & $\begin{array}{l}\text { Estudo primário } \\
\text { prospetivo }\end{array}$ & $\begin{array}{l}\text { Participantes- } 33 \text { pacientes com DP. } \\
\text { Intervenções- Exercícios terapêuticos de força, resistência } \\
\text { e flexibilidade, efetuados durante } 60 \text { minutos, } 3 \text { vezes por } \\
\text { semana ao longo de } 12 \text { semanas no domicílio. } \\
\text { Resultados- Melhorias nas atividades de vida diária (AVD), } \\
\text { na função motora e na mobilidade. }\end{array}$ \\
\hline
\end{tabular}




\begin{tabular}{|c|c|c|}
\hline Estudos/Autores/País & Tipo de Estudo & Participantes/Intervenções/Evidencia alcançada \\
\hline $\begin{array}{l}\text { E2 } \\
\text { Ferrazzoli et al. (2018). } \\
\text { Itália }\end{array}$ & $\begin{array}{l}\text { Estudo clínico } \\
\text { prospetivo e } \\
\text { randomizado. }\end{array}$ & $\begin{array}{l}\text { Participantes-186 pacientes com DP, integrando } 93 \text { o } \\
\text { grupo de controlo (GC)e } 93 \text { o grupo experimental. } \\
\text { Intervenções- Programa de Reabilitação multidisciplinar - } \\
1 \text { hora por semana durante } 10 \text { semanas com } 2 \text { avaliações. } \\
\text { Resultados- Melhoria significativa na mobilidade, nas AVD, } \\
\text { bem-estar emocional, suporte social, cognição, } \\
\text { comunicação e qualidade de vida, apenas na última } \\
\text { avaliação. }\end{array}$ \\
\hline E3 & $\begin{array}{l}\text { Estudo clínico } \\
\text { prospetivo e }\end{array}$ & $\begin{array}{l}\text { Participantes-126 pacientes com DP, integrando } 63 \text { o } \\
\text { grupo experimental (GE) e } 63 \text { o GC. }\end{array}$ \\
\hline
\end{tabular}

Kwok et al. (2017). randomizado.

China

Intervenções- No GE, treino de força e resistência, associado a sessões de yoga, 90 minutos por semana durante 8 semanas. No GC, uma sessão semanal de 60 minutos de treino de força e resistência muscular.

Resultados- Melhorias na flexibilidade, marcha e equilíbrio, maior relaxamento corporal e função mental apenas no GE.

\section{E4}

Bekkers et al. (2014).

Estados Unidos da

América

\section{E5}

Liao et al. (2015).

China
E6

Silva et al.

Brasil.

E7

Shen et al. (2015).

Estudo clínico prospetivo e randomizado.

Estudo clínico prospetivo e randomizado

China
Estudo prospetivo randomizado controlado

Estudo clínico quantitativo estratificado, randomizado controlado.
Participantes- 26 pacientes com DP com história de quedas; 13 pacientes no GE e 13 no GC;

Intervenções- exercícios terapêuticos de informação propriocetiva no controlo postural (vibração do tendão de Aquiles)

Resultados- maior equilíbrio corporal médio-lateral e ântero-posterior, no GE.

Participantes- 36 pacientes com DP divididos em 3 grupos:

-12 no grupo 1;12 no grupo 2; e 12 no GC.

Intervenções- G1 - Efeito da Realidade Virtual (estimulação visual e auditiva);G2- Exercícios terapêuticos clássicos;GC-Sem intervenção.

Resultados- Melhorias a contornar os obstáculos na postura e na velocidade de movimento - G1.Melhorias no equilíbrio dinâmico e organização sensorial no G2.

Participantes-18 pacientes com DP (8 no GE e 10 no GC).

Intervenções- Foram realizadas 15 sessões de reabilitação física, cada uma com a duração de 40 minutos e 2 vezes por semana, nos dois grupos. No GE, foi associada exercitação mental, com a duração de 15 minutos por sessão (sequencia de passos).

Resultados- Melhor avaliação no Índice de marcha dinâmica, melhoria da mobilidade funcional com redução do risco de queda e menor tempo de execução real do passo no GE.

Participantes- 51 pacientes com DP (26 no GE e 25 no GC).

Intervenções- programa tecnológico inovador (computorizado) para o equilíbrio corporal e treino de marcha no GE (duração de 12 semanas, em que nas primeiras $8 \mathrm{com}$ uma frequência de 3 sessões por semana em laboratório e nas últimas 4 semanas em contexto domiciliário, com 5 dias de sessões semanais).

GC- Programa de fortalecimento muscular.

Resultados- Diminuiu o número de quedas no GE aos 3, 6 e 15 meses após o início da intervenção; aumento do comprimento do passo e no apoio do pé.

Aumento da velocidade da marcha nos dois grupos. 


\section{Discussão dos Resultados}

Os estudos incluídos nesta revisão, como se constatou, são maioritariamente clínicos, prospetivos e randomizados controlados, com diversos instrumentos de avaliação, porém permitindo a avaliação da eficácia de intervenções dos programas de Reabilitação em pessoas portadoras da DP. A análise individualizada dos estudos mostrou alguma homogeneidade no desenho dos estudos, mas uma grande heterogeneidade relativamente ao número da amostra, aos objetivos formulados, às variáveis estudadas e ao tipo de intervenção aplicada. Esta heterogeneidade impossibilitou a realização de metanálise, o que viria aumentar a objetividade e validade dos achados.

A amostra dos estudos referidos era composta por doentes com DP, em estádios da doença diferenciados (III, IV e V) e as idades oscilaram entre os 48 e os 77 anos. Globalmente, os estudos apresentavam GE e GC (sem intervenção ou com intervenção diferenciada). Utilizaram maioritariamente, como método de avaliação da eficácia dos programas de Reabilitação e questionários, aplicados em diferentes momentos. Foram feitos antes e depois da intervenção e em alguns casos às $2^{\mathrm{a}}, 4^{\mathrm{a}}, 8^{\mathrm{a}}, 12^{\mathrm{a}}$ e $18^{\mathrm{a}}$ semanas após intervenção. Veja-se a este propósito que a eficácia das intervenções se diferenciou pela positiva tendo por base o período temporal em que decorreram os programas. O E1 de Santos et al. (2012) mostrou melhores resultados no desempenho das AVD, na mobilidade e equilíbrio apenas na avaliação efetuada à $12^{\underline{a}}$ semana de intervenção. Estes dados estão alinhados com os de Lizon, Cholewa, \& Uher (2017),ao enfatizar as melhorias verificadas sobretudo no autocuidado e na mobilidade.

Similarmente o E2 de Ferrazzoli et al (2018), revelou que após 2 semanas de implementação do $\mathrm{PR}$, não se encontraram melhorias nas atividades pretendidas, contudo à $10^{a}$ semana, a avaliação traduziu resultados bem mais satisfatórios, sendo as diferenças estatísticas significativas $(p<0.001)$ nos resultados do Global Index Score (GBI). As melhorias verificaram-se globalmente na mobilidade, nas AVD, no bem-estar emocional, suporte social, e cognição. O E5 de Liao, Yang, Cheng, Wu, Fuh \& Wang (2015) mostrou também que o equilíbrio dinâmico e a organização sensorial foram dimensões que melhoraram de forma significativa entre a $4^{0}$ e a $8^{\text {a }}$ semana de intervenção. O E7 de Shen e Mak (2015), vem também reforçar a pertinência dos períodos de realização dos programas, ao concluírem que o número de quedas no GE diminui de forma continuada, aos 3, 6 e 15 meses após o início das intervenções. Luz e Coronago (2017) afirmam que os programas de reabilitação continuadados demonstram níveis de eficácia superiores quando comparados com outros mais curtos sobretudo ao nível da postura, da mobilidade e na prevenção das quedas.

A análise do tipo de intervenções e programas instituídos demonstra que na generalidade dos estudos existiu a implementação de exercícios terapêuticos direcionados à dimensão física como o treino de força, resistência e flexibilidade, treino de equilíbrio corporal e ainda o treino de marcha. Porém, é de realçar aspetos inovadores que resultaram da associação de outras metodologias de intervenção e que se revelaram muito positivos. Veja-se o caso de E3 de Kwok, Kwan, Auyeung, Mok \& Chan (2017) que implementou num dos grupos uma sessão semanal de Yoga de 90 minutos durante 8 semanas, associado ao treino de força e resistência muscular. Os benefícios encontrados neste grupo foram uma melhoria na flexibilidade, marcha e equilíbrio, maior relaxamento corporal e bem-estar holístico. Segundo os autores, a estimulação da perceção corporal em termos de mobilidade (reforçada pelo Yoga) melhorou sintomas motores, diminuiu instabilidade postural e aumentou a capacidade funcional das pessoas com DP, sugerindo a sua implementação como parte integrante de um Plano de Reabilitação neste tipo de doentes. Estes dados são parcialmente corroborados pelos de Souza et al. (2017) ao demonstrarem que as sessões de yoga podem ser tão eficazes quanto os treinos de alongamento e resistência para quem tem Parkinson. Porém, no que diz respeito à redução de ansiedade á depressão e bem-estar, o yoga teve melhor desempenho que os demais exercícios. 
O E4 de Bekkers et al. (2014) dá também especial ênfase ao efeito terapêutico da informação propriocetiva no controlo postural em doentes com Parkinson, com história prévia de quedas. Os resultados demonstraram diferenças estatisticamente significativas no grupo experimental (valor de $\mathrm{p}=0.043$ ), comparado com o grupo de controlo, no que concerne ao maior equilíbrio corporal médio-lateral do primeiro grupo. Não obstante, em relação à direção antero-posterior, os resultados demonstram diferenças estatisticamente significativas no equilíbrio corporal em superfícies instáveis e na presença de vibração do tendão de Aquiles em comparação com a presença sobre superfícies estáveis (valor de $p<0.001$ ). Resultados semelhantes são apresentados por Nielson et al. (2018) ao verificarem que o treinamento que inclua exercícios de proprioceção, melhora significativamente a postura e a capacidade de manter o equilíbrio durante condições de instabilidade.

O estudo 5 realizado por Liao, Yang, Cheng, Wu, Fuh e Wang (2015) pretendia avaliar o efeito provocado pela Realidade Virtual (através de estimulação visual e auditiva), na forma como os doentes com Parkinson ultrapassam determinados obstáculos, na marcha e no equilíbrio corporal. Em relação a contornar os obstáculos, o primeiro grupo apresentou melhorias estatisticamente significativas (valor de $p<0.05$ ), comparado com o grupo de controlo. Para além deste resultado, o grupo que teve acesso às sessões com Realidade Virtual exibiu melhores índices do que os participantes com programa de exercícios terapêuticos, nomeadamente com alterações estatisticamente significativas na postura e na velocidade de movimento $(p<0.05)$. Este facto foi confirmado por um estudo desenvolvido por Tan et al. (2014), que concluiu que o tratamento com realidade virtual, melhorou a qualidade de vida dos idosos com doença de Parkinson, com especial ênfase para a percepção de qualidade de vida relacionada com o bem-estar emocional e estigma, seguidos da mobilidade e cognição. Sugere ainda, que o enfrentamento da doença de Parkinson deve contemplar, além de seus sinais e sintomas, aspectos relacionados à qualidade de vida, a fim de não subestimar a complexidade do sujeito e a repercussão subjetiva da doença e/ ou tratamento.

O E6 realizado por Silva et al (2019) pretendia avaliar os efeitos da exercitação mental associada à reabilitação física motora sobre a marcha e o risco de queda em pessoas com DP. Os resultados demonstraram diferenças estatisticamente significativas do grupo experimental $(p<0.05)$ na avaliação do Índice de marcha dinâmica, considerando os autores que após a intervenção (com exercitação mental) houve melhoria da mobilidade funcional com redução do risco de queda dos doentes. Consideram ainda, que uma melhor aprendizagem motora é de grande importância para os doentes, ao permitir o armazenamento e consolidação de informações motoras na memória de longo prazo, promovendo-se igualmente o aumento da precisão espacial e temporal do movimento. De forma similar o E7 de Shen e Mak (2015), pretendia avaliar os efeitos de um programa tecnológico para o equilíbrio corporal e de treino de marcha na redução de quedas em doentes com DP. Os resultados demonstraram que o grupo submetido ao programa tecnológico registou um aumento significativo do comprimento do passo e no tempo de apoio do pé. Apesar do aumento na velocidade da marcha verificado nos dois grupos, os autores consideram importante a incorporação de novos sistemas tecnológicos, uma vez que estes podem contribuir para o incremento de mais e melhor inovação científica no domínio da Reabilitação em doentes com Parkinson. Estes resultados constituem-se como reforço e validação dos já sugeridos por diferentes estudos, desde a APDP, (2019), Santos, (2018) e Tan et al. (2014), ao recomendarem a implementação de programas multi e interdisciplinares nos pacientes com esta patologia.

\section{Conclusões}

A evidência destes estudos primários sugere uma eficácia efetiva dos programas de Reabilitação, com relevância estatística significativa, em diferentes dimensões. As melhorias observadas, foram além do foco principal do nosso estudo. Constatámos que revelaram melhoria da mobilidade (todos os estudos); diminuição e/ou prevenção do risco de quedas (E5, E6 e E7); melhoria nas AVD (E1 e E2) e melhoria do bem-estar dos sujeitos (E1, E2 e E3). 
Um outro aspeto que consideramos relevante foi a introdução de novas estratégias de intervenção nos programas implementados, revelando-se estas também eficazes nas melhorias pretendidas. Nestas intervenções estão incluídas as sessões de Yoga (E3), o efeito terapêutico da informação propriocetiva no controlo postural (E4), o recurso á realidade virtual (através de estimulação visual e auditiva) (E5), os efeitos da exercitação mental associada à reabilitação física motora $(E 6)$ e ainda o recurso a programas tecnológicos (E7).

Igualmente interessante, é o local de realização dos programas: de facto a sugestão de programas de Reabilitação domiciliários dirigidos às pessoas/ famílias, possibilita a implementação de planos diferenciados e ajustados às condições circunstanciais do doente com Parkinson fora do ambiente hospitalar.

Foram consideradas limitações desta pesquisa: o número reduzido de estudos; as diferenças metodológicas seguidas, dificultando a comparabilidade e generalização de resultados; a limitação do idioma escrito (português, espanhol e Inglês) sentida na tradução dos descritores; a inexistência de programas de Reabilitação implementados apenas por ER. Pese embora, a complexidade e diversidade dos estudos apresentados (mas sempre respondendo às questões de investigação), consideramo-los importantes ao permitir que novas atualizações de cariz técnico-científico possam ser equacionadas e integradas nos programas de Reabilitação para aplicação em contexto de cuidados.

Como implicações, reforçamos a necessidade de promover mais Investigação sobre o tema em análise, com validação e avaliação dos programas de Reabilitação que demonstrem eficácia na melhoria da mobilidade, na prevenção e na diminuição do risco de queda em doentes com Parkinson. Propomos ainda estudos com metodologias semelhantes de modo a aumentar a capacidade de comparação e possibilidade de criação de recomendações das evidências clínicas. Quanto às implicações para a prática, recomendamos a elaboração de programas de Reabilitação estruturados que integrem um misto de intervenções "clássicas" e novas abordagens terapêuticas (acima explicitadas) pois parece ser evidente a obtenção de resultados

O incremento da produção científica na área específica da Enfermagem de Reabilitação é um processo desafiador, por permitir adquirir e desenvolver competências e habilidades fundamentais na sua prática profissional quotidiana.

\section{Referências}

Associação Portuguesa de Doentes de Parkinson. (2019). Manual para pessoas com Parkinson. https://apdpk298646906.files.wordpress.com/2019/11/parkinson-manual_pt.pdf

Barbosa, E. R., \& Melo, L. M. (2007). Importância das manifestações não motoras da doença de Parkinson. Revista Neurociências, 15, 51-60.

http://www.revistaneurociencias.com.br/edicoes/2007/RN\%2015\%2001/Pages\%20from\%20R N\%2015\%2001-8.pdf

Bekkers, E. M. J., Dockx, K., Heremans, E., Vercrruysse, S., Verschueren, S. M. P., Mirelman, A., \& Nieuwboer, A. (2014). The contribution of proprioceptive information to postural control in elderly and patients with Parkinson's disease with a history of falls. Frontiers in Human Neuroscience, 8, 1-9. doi: doi.org/10.3389/fnhum.2014.00939

Carneiro, A. V. (2008). Como avaliar a investigação clínica: O exemplo da avaliação crítica de um ensaio clínico. Jornal Português de Gastrenterologia, 15(1), 30-36. http://www.scielo.mec.pt/scielo.php?script=sci_arttext\&pid=S0872$81782008000100007 \& \operatorname{lng}=$ pt\&tlng=pt

Ferrazzoli, D., Ortelli, P., Zivi, I., Veronica, C., Urso, E., Ghilardi, M., ... Frazzitta, G. (2018). Efficacy of intensive multidisciplinary rehabilitation in Parkinson's disease: A randomised controlled study. Journal of Neurology, Neurosurgery and Psychiatry, 89, 828-835. doi: 10.1136/jnnp-2017-316437

Instituto Nacional de Estatística. (2019). Causas de morte 2017. Lisboa, Portugal: INE. 
Joanna Briggs Institute (2015). The Joanna Briggs Institute reviewers' manual 2015: Methodology for JBI scoping reviews. http://joannabriggs.org/assets/docs/sumari/ReviewersManual_Methodology-for-JBI-Scoping--Reviews_2015_v2.pdf

Kwok, J. Y. Y., Kwan, J. C. Y. K., Auyeung, M., Mok, V. C. T., \& Chan, H. Y. L. (2017). The effects of yoga versus stretching and resistance training exercises on psychological distress for people with mild-to-moderate Parkinson's disease: Study protocol for a randomized controlled trial. Trials, 18, 1-13. doi: 10.1186/s13063-017-2223-x

Liao, Y. Y., Yang, Y. R., Cheng, S. J., Wu, Y. R., Fuh, J. L., \& Wang, R. Y. (2015). Virtual realitybased training to improve obstacle-crossing performance and dynamic balance in patients with Parkinson's disease. Neurorehabilitation and Neural Repair, 29(7). doi: $10.1177 / 1545968314562111$

Lizon, K., Cholewa, J., \& Uher, I. (2017). Influence of physical rehabilitation on social networks and quality of life among people with Parkinson's disease. Journal of Physical Education and Health, 10, 35-42. http://cejsh.icm.edu.pl/cejsh/element/bwmeta1.element.desklighte98e13fc-20ea-4078-b8a7-3a7471dbb548

Luz, K. P. S., \& Coronago, V. M. M. O. (2017). A doença de Parkinson na pessoa idosa e a relação com sua qualidade de vida. Revista Multidisciplinar e de Psicologia, 11(35), 116-136. doi: 10.14295/idonline.v11i35.720

Martins, R., Figueiredo, A., Andrade, A., Albuquerque, C., \& Martins, C. (2018). Investimento na vida pessoal em idosos institucionalizados. Revista de Investigação \& Inovação em Saúde, 1(1), 7-15. doi: 10.37914/riis.v1i1.24

Nielsen, M. B., Santos, Y. F., Gering, E., \& Sant'Ana, J. E. (2018). Sinais prodrómicos na doença de Parkinson: Prevalência da constipação intestinal.

http://revista.redeunida.org.br/ojs/index.php/cadernos-educacao-saudefisioter/article/view/1944

Rocha, L. S. O., Mineshita, N. H., Sobral, L. L., Santos, M. C. S., \& Rocha, R. S. B. (2017). Influence of global postural reeducation method on respiratory muscle strenght and parkinsonian quality of life. Manual Therapy, Posturology \& Rehabilitation Journal, 15. doi:10.17784/mtprehabjournal.2017.15.504

Santos, A. R. S. (2018). Sintomas não motores na doença de Parkinson: Estudo qualitativo exploratório [Dissertação de mestrado]. Universidade de Lisboa, Portugal.

Santos, V. V., Araújo, M. A., Nascimento, O. J. M., Guimarães, F. S., Orsini, M., \& Freitas, M. R. G. (2012). Effects of a physical therapy home-based exercise program for Parkinson's disease. Fisioterapia e Movimento, 4(25), 709-715. doi: 10.1590/S0103-51502012000400003

Shen, X., \& Mak, M. K. Y. (2015). Technology-assisted balance and gait training reduces falls in patients with Parkinson's disease: A randomized controlled trial with 12-month follow-up. Neurorehabilitation and Neural Repair, 29, 103-111. doi: 10.1177/1545968314537559

Silva, L. P., Duarte, M. P. S., Souza, C. C. B., Lins, C. C. S. A., Coriolano, M. G. W. S., \& Lins, O. G. (2019). Efeitos da prática mental associada à fisioterapia motora sobre a marcha e o risco de quedas na doença de Parkinson: Estudo piloto. Fisioterapia e Pesquisa, 2, 112-119. doi: $10.1590 / 1809-2950 / 17012926022019$

Sousa, A.S., Lopes, K. L., Fragnani, S. G., Nery, T., Werner, E., Bezerra, P. P. (2017) Physiotherapy associated with yoga and musicotherapy in parkinson's disease: clinical trial. Rev Bras de Neurol. 53(3): 31-40, 2017 https://docs.bvsalud.org/biblioref/2017/12/876875/rbn-533-4-fisioterapia-associada-ayoga.pdf

Tan, S. B., Williams, A. F., \& Kelly, D. (2014). Effectiveness of multidisciplinar interventions to improve the quality of life for people with Parkinson's disease: A systematic review. International Journal of Nursing Studies, 51(1), 166-174. doi: 10.1016/j.ijnurstu.2013.03.009 